\title{
Drawable Region of the Generalized Log Aesthetic Curves
}

\author{
R. U. Gobithaasan, ${ }^{1}$ R. Karpagavalli, ${ }^{1}$ and Kenjiro T. Miura ${ }^{2}$ \\ ${ }^{1}$ Department of Mathematics, FST, Universiti Malaysia Terengganu, 21030 Kuala Terengganu, Malaysia \\ ${ }^{2}$ Graduate School of Science \& Technology, Shizuoka University, Shizuoka 432-8561, Japan \\ Correspondence should be addressed to R. U. Gobithaasan; gr@umt.edu.my
}

Received 3 June 2013; Accepted 16 September 2013

Academic Editor: Kazutake Komori

Copyright (C) 2013 R. U. Gobithaasan et al. This is an open access article distributed under the Creative Commons Attribution License, which permits unrestricted use, distribution, and reproduction in any medium, provided the original work is properly cited.

\begin{abstract}
The main characteristic of visually pleasing curves used for product design is a monotonic curvature profile. Recently, a planar curve called Generalized Log Aesthetic Curve (GLAC) has been extended from the Log Aesthetic Curve (LAC), and it has an additional shape parameter, $\nu$. This curve preserves the monotonicity of curvature and is said to produce visually pleasing curves. This paper delves on the drawable region of the GLAC segment which indicates the probable solutions of shape parameters from given interpolating points and the direction of travel at those points. The first section reviews the formulation of GLAC and its related bounds. The section describes the algorithm for identifying the drawable region. It is followed by the section describing how small changes of $v$ widen the drawable boundaries. The final section discusses the superiority of GLAC compared to LAC for use in industrial product design.
\end{abstract}

\section{Introduction}

The importance of aesthetic shapes to design industrial products leads to the studies of planar aesthetic curves [1]. High quality curves are also known as fair curves, minimal energy curves, aesthetic curves, and so forth. The main characteristic of these curves is that it has a monotonic curvature profile. The mathematical term used to describe planar curves with monotonic (either increase or decrease) curvature is called a spiral [2]. A curve is categorized as fair if its curvature plot consists of relatively minimum number of monotone pieces.

In 1999, Harada et al. presented a quantitative method of investigating curves used in automobile design which is called Logarithmic Distribution Diagram of Curvature (LDDC). They defined aesthetic curves as curves with a constant LDDC gradient (denoted as $\alpha$ ). Consequently in 2003, Kanaya et al. simplified the formulation of LDDC to Logarithmic Curvature Graph (LCG). In 2005, Miura analyzed the features of aesthetic curves and derived a general formula of aesthetic curves called Log Aesthetic Curve (LAC). Yoshida and Saito [3] further investigated LAC to identify the overall shapes of LAC. They proposed a method to draw LAC curve segment interactively by using two endpoints and their respective tangent vectors. In 2009, Levien and Sequin stated that the LA curve is the most promising curve for aesthetic design. In 2012, Yoshida and Saito derived a method to render the drawable boundary for LAC segments.

The number of research papers produced since the introduction of LAC has been increasing exponentially such as the reformulation of $2 \mathrm{D}$ and $3 \mathrm{D}$ LAC in the form of variational principal [4], analytic representation of LA curve using Incomplete Gamma function for efficient computation [5], and the formulation of G2 LA spline for automobile design application [6].

Recently, a planar curve called Generalized Log Aesthetic Curve (GLAC) has been developed by extending the formulation of Generalized Cornu Spiral (GCS) [7] in a similar manner to the LAC [8]. The family of GLAC comprises planar curves of high quality such as GCS, LAC, clothoid, Nielsen's spiral, logarithmic spiral, circle involute, and so forth. The GLAC segment has an additional parameter (denoted as $\nu$ ) to determine its shape as compared to GCS and LAC segment. Hence, an extra constraint can be satisfied when shaping the GLAC segment. Recent advancements of GLAC include the 
TABLE 1: The lower bound and upper bound for $s$.

\begin{tabular}{|c|c|c|c|c|c|c|}
\hline \multicolumn{7}{|c|}{ Arc length } \\
\hline & \multicolumn{2}{|c|}{$v<0(\nu \neq-1)$} & \multicolumn{2}{|c|}{$v>0$} & \multicolumn{2}{|c|}{$v=-1$} \\
\hline & LB & UB & LB & UB & LB & UB \\
\hline$\alpha<0$ & - & $\frac{(-\gamma)^{-\alpha}-1}{\Lambda \alpha}$ & - & $-\frac{1}{\Lambda \alpha}$ & - & 0 \\
\hline$\alpha=0$ & - & $-\frac{\log [-\nu]}{\Lambda}$ & - & - & - & 0 \\
\hline$\alpha>0$ & $-\frac{1}{\Lambda \alpha}$ & $\frac{(-\gamma)^{-\alpha}-1}{\Lambda \alpha}$ & $-\frac{1}{\Lambda \alpha}$ & - & $-\frac{1}{\Lambda \alpha}$ & 0 \\
\hline
\end{tabular}

extension of spatial GLAC [9] and the shape analysis of GLAC [10].

Interactive control of GLAC segment has been developed similar to interactive LAC segment [11]. However, it is necessary to analyze the drawable region of interactive GLAC segment so that the users are aware of the flexibility of GLAC for shape design. The identification of drawable region of GLAC is similar to the method employed for LAC [12]. However, a slight modification has been carried out to determine the shape parameter $\Lambda$. We modify the arc length instead of modifying the $\theta_{d}$ directly. We will modify arc length in the range of $0<s<\infty$, which will give $\theta_{d}$ such that $0<\theta_{d}<\pi$.

1.1. The Formulation of GLAC. GLAC is derived via the curve synthesis process where a formulation of a curve is derived from defined curvature function. The advantage of GLAC is that it has an extra shape parameter $(\nu)$ which can be used to dictate the curvature radius $(\rho)$ at the starting point of the GLAC. GLAC becomes LAC when $\nu$ becomes 0 . The LCG and its gradient function, $\lambda(s)$ are derived for GLAC as follows [8]:

$$
\begin{aligned}
& \operatorname{LCG}_{\mathrm{GLAC}}(s) \\
& =\left\{\log \left[\frac{1}{(\Lambda \alpha s+1)^{-1 / \alpha}+v}\right],\right. \\
& \left.\log \left[\frac{1}{\Lambda}(\Lambda \alpha s+1)\left(1+\nu(\Lambda \alpha s+1)^{1 / \alpha}\right)\right]\right\}, \\
& \lambda(s)_{\mathrm{GLAC}}=\alpha+\nu(\Lambda \alpha s+1)^{1 / \alpha}(1+\alpha) .
\end{aligned}
$$

Arc length and tangential angle function of GLAC are shown below, respectively:

$$
s_{\mathrm{GLAC}}(\rho)= \begin{cases}\frac{1}{\Lambda} \log \left[\frac{1}{\rho^{-1}-\nu}\right] & \text { if } \alpha=0, \\ \frac{1}{\Lambda \alpha}\left(\left(\rho^{-1}-\nu\right)^{-\alpha}-1\right) & \text { otherwise, }\end{cases}
$$

$$
\begin{aligned}
& \theta_{\mathrm{GLAC}}(s) \text { if } \alpha=0, \\
& \quad= \begin{cases}\frac{1}{\Lambda}\left(1-e^{-\Lambda s}\right)+v s & \text { if } \alpha=1, \\
\frac{1}{\Lambda} \log [\Lambda s+1]+v s & \text { otherwise. } \\
\frac{1}{\Lambda(\alpha-1)}\left((\Lambda \alpha s+1)^{(\alpha-1) / \alpha}-1\right)+v s\end{cases}
\end{aligned}
$$

The parametric form of GLAC is

$$
C_{\mathrm{GLAC}}(s)=\left\{\int_{0}^{s} \cos \left[\theta_{\mathrm{GLAC}}(u)\right] d u, \int_{0}^{s} \sin \left[\theta_{\mathrm{GLAC}}(u)\right] d u\right\},
$$

where $\{\Lambda, \alpha, \nu\} \in \mathbb{R}$ are the variables that can be used to shape the GLAC segment.

1.2. The Bounds of GLAC. The bounds are derived for both arc length and tangential angle function of GLACwhich are depicted in Table 1 and Table 2 respectively. For the case of $\nu=0$, the bounds of GLAC are similar to the bounds of LAC as stated in [10].

\section{Drawable Region of GLAC}

Interactive control of GLAC segment can be developed either by stating the value of $\nu$ or allowing the optimization techique to find a suitable value for $\nu$. It is possible to construct the drawable region for interactive GLAC if the designer chooses to set the value for $\nu$. The analysis of the drawable region can then be used to identify all possible solutions that exist for a given input.

2.1. The Method. The method used is similar to LAC where three control points will be placed in a rectangle of corners $( \pm 1, \pm 1)$. Values of $\alpha<0$ are used to construct the drawable region for the case of $\nu>0$. For the case of $-1<v<0$, there is no restriction for $\alpha$. Figure 1 illustrates the setup of control points in order to obtain the drawable region of GLAC.

Let points $P_{0}, P_{1}$, and $P_{2}$ be the control points for drawing a GLAC segment. The points $P_{0}$ and $P_{2}$ are placed at $(-1,0)$ and $(1,0)$. The point $P_{1}$ is the point on the drawable boundary in the rectangle. To note, $P_{1}$ is unknown and will be determined by the tangential angle $\theta_{d}$ on the curve. GLAC 
TABLE 2: The lower bound and upper bound for $\theta$.

\begin{tabular}{|c|c|c|c|c|c|c|}
\hline \multicolumn{7}{|c|}{ Tangential angle } \\
\hline & \multicolumn{2}{|c|}{$v<0(\nu \neq-1)$} & \multicolumn{2}{|c|}{$v>0$} & \multicolumn{2}{|c|}{$\nu=-1$} \\
\hline & LB & UB & LB & UB & LB & UB \\
\hline$\alpha<0$ & - & A & - & $\mathrm{B}$ & - & 0 \\
\hline$\alpha=0$ & - & $\frac{1}{\Lambda}(1+\nu-\nu \log [-\nu])$ & - & - & - & 0 \\
\hline $0<\alpha<1$ & - & A & - & - & - & 0 \\
\hline$\alpha=1$ & - & $-\frac{1}{\Lambda}(1+\nu+\log [-\nu])$ & - & - & - & 0 \\
\hline$\alpha>1$ & $\mathrm{~B}$ & A & $\mathrm{B}$ & - & $\frac{1}{\Lambda \alpha(1-\alpha)}$ & 0 \\
\hline
\end{tabular}

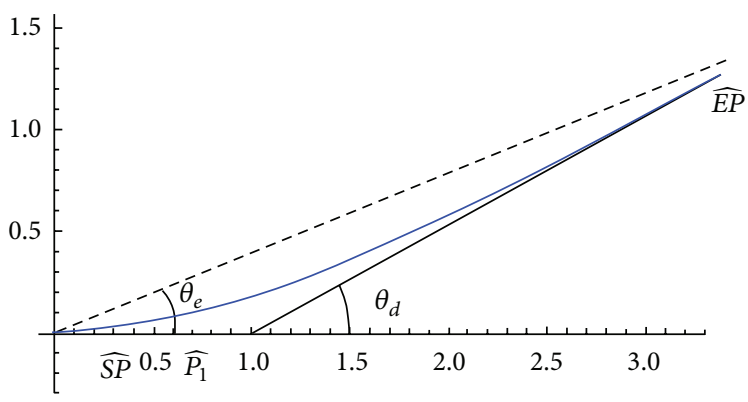

(a) GLAC segment with new endpoints

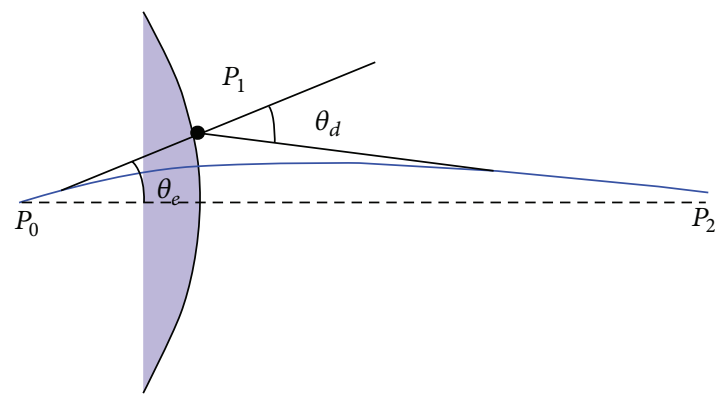

(b) GLAC segment and a drawable boundary

FIgURE 1: A drawable region of interactive GLAC segment.

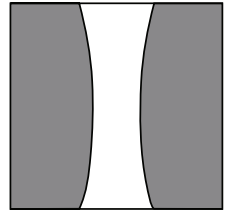

(a) $\alpha=-3$

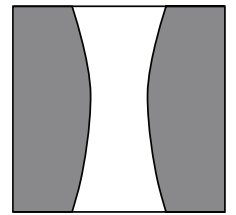

(b) $\alpha=-2$

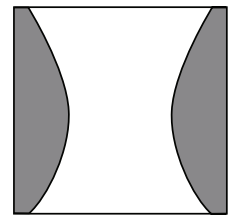

(h) $\alpha=0.8$

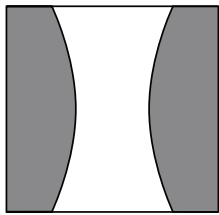

(c) $\alpha=-1$

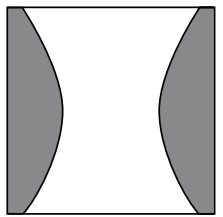

(i) $\alpha=1$

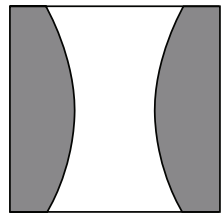

(d) $\alpha=-0.5$

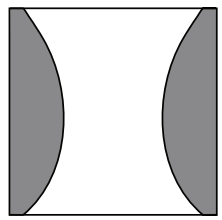

(j) $\alpha=1.1$

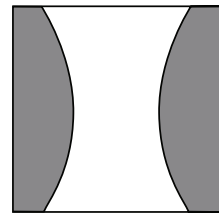

(e) $\alpha=-0.1$

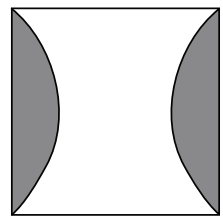

(k) $\alpha=2$

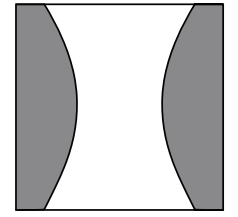

(f) $\alpha=0$

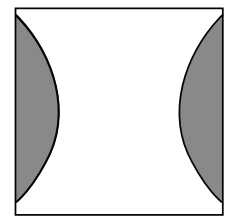

(l) $\alpha=3$

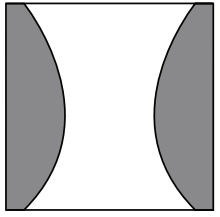

(g) $\alpha=0.5$

FIgURE 2: The drawable region of GLAC when $v=-0.3$.

segment with tangential angle from 0 to $\theta_{d}$ is used for all $\alpha$. Point $P_{1}$ can be found on the rectangle by performing transformation. By changing the arc length from 0 to a large number, the points of $P_{1}$ will form the drawable boundary. Note that we are modifying arc length to obtain $\theta_{d}$ in the range of $0<\theta_{d}<\pi$. Hence, by applying reflection on the $x$-axis and $y$-axis, a complete drawable boundary can be found.

2.2. The Algorithm. The objective of this algorithm (Algorithm 1) is to determine the second control point through the formulation of GLAC curve segment and by plotting it on the rectangle.

\section{Analysis Result}

Figures 2, 3, 4, 5, 6, 7, 8, 9, 10, and 11 show the obtained result for the drawable region of the interactive GLAC segment depending on $\alpha$ and $\nu$. Figures 2, 3, 4, 5, 6, and 7 show the pattern of how the decrease and increase of $v$ affects the drawable region. Figures 8, 9, 10, and 11 show the drawable region for $\alpha=-1,0,1,2$ as $v$ increases. The white region 
Remark: Control points $\left\{P_{0}, P_{2}\right\}$ are given with a preferred $\alpha$. Via GLAC function, second control point is determined. New points are transformed to the originals and plotted. $s$ is modified from 0 to a large number such that $\theta_{d}$ is in range of $0<\theta_{d}<\pi$ to get the complete drawable boundary.

Input: $P_{0}(-1,0), P_{2}(1,0), \alpha, v, s$.

Output: Complete drawable boundary of interactive GLAC.

Begin

Step 1. If $-1<v<0$ then

if $\alpha=0$ then

else

$$
\Lambda \leftarrow \frac{-\log [-\nu]}{s} .
$$

else if $\nu>0$ and $\alpha<0$ then

$$
\Lambda \leftarrow \frac{(-\nu)^{-\alpha}-1}{s \alpha} .
$$

$$
\Lambda \leftarrow \frac{-1}{s \alpha} .
$$

Step 2. Determine $\widehat{S P}\left(x_{0}, y_{0}\right)$ and $\widehat{E P}\left(x_{2}, y_{2}\right)$ using (3).

Step 3. Determine scaling factor $r \leftarrow \frac{\left\|P_{2} P_{0}\right\|}{\|\widehat{S P} \widehat{E P}\|}$ and angle $\theta_{e} \leftarrow \cos ^{-1}\left[\frac{x_{2}}{\sqrt{x_{2}^{2}+y_{2}^{2}}}\right]$.

Step 4. Determine $\widehat{P_{1}}$ using the tangent line of both end points.

Step 5. Scale points to $r$, reflect through $x$-axis, rotate to $-\theta_{e}$ and translate to $\left\{P_{0}, P_{2}\right\}$.

Step 6. Plot point $P_{1}$.

Step 7. Modify $s$ from 0 to a large number and repeat Step 1. to 6.

Step 8. Reflect points via $x$-axis and $y$-axis.

Step 9. Output.

End.

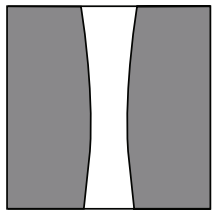

(a) $\alpha=-3$

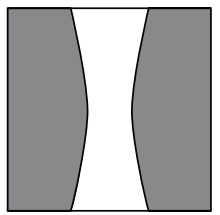

(b) $\alpha=-2$

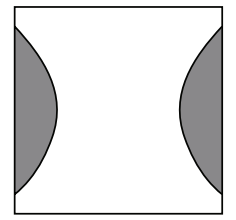

(h) $\alpha=0.8$

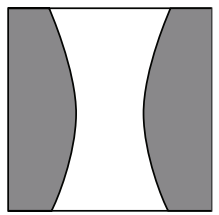

(c) $\alpha=-1$

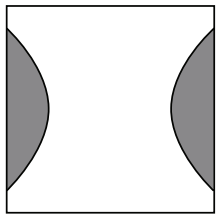

(i) $\alpha=1$

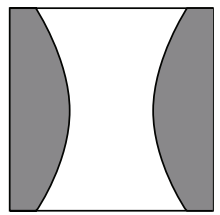

(d) $\alpha=-0.5$

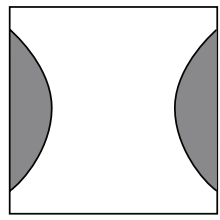

(j) $\alpha=1.1$

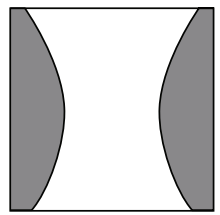

(e) $\alpha=-0.1$

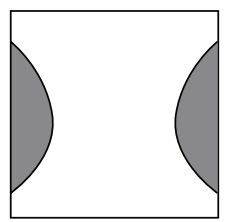

(k) $\alpha=2$

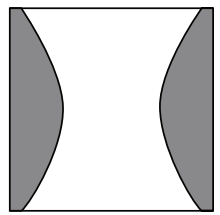

(f) $\alpha=0$

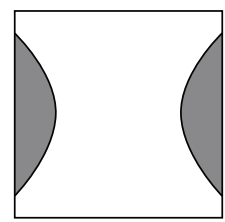

(l) $\alpha=3$

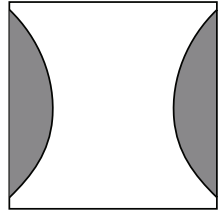

(g) $\alpha=0.5$

FIGURE 3: The drawable region of GLAC when $v=-0.1$.

implies the drawable region of GLAC segment, while the gray region indicates no solution. Hence, more probable GLAC shapes can be drawn when the white region widens.

The drawable region of GLAC shows a better result when $-1<v<0$ compared to when $v>0$. This can be observed in Figure 4 where the drawable boundaries are larger compared to Figure 7. When $v$ is positive (small value, e.g., when $v=$ 0.01 ) and $-1<\alpha<0$, the drawable region is quite larger as compared to when $-1<v<0$. However, when $v$ is positive, the drawable region gets smaller for all other $\alpha$. This implies that the shape variable $v$ plays a major role in dictating the drawable region effectively. The drawable regions can be expended remarkably with a small change of $\nu$. Generally, $-1<v<0$ gives a wider drawable region when $\alpha>1$ and $\alpha<-1$. We can note that when $\alpha=2$, the drawable region is very large compared to LAC. LAC has regions that have no solutions, whereas GLAC provides the solution.

According to Yoshida and Saito [12], there are cases where the LAC segment is not drawable even when the second control point is within the drawable boundary. GLAC faces a similar situation where the second control point is within the drawable region yet the curve segment is not drawable. 


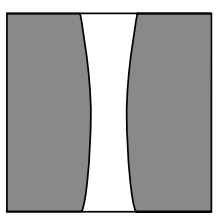

(a) $\alpha=-3$

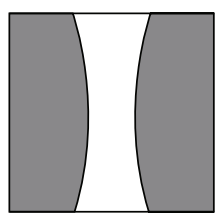

(b) $\alpha=-2$

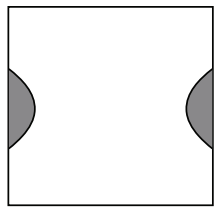

(h) $\alpha=0.8$

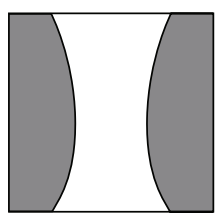

(c) $\alpha=-1$

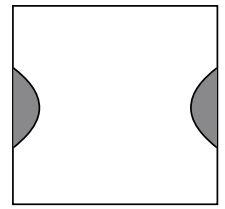

(i) $\alpha=1$

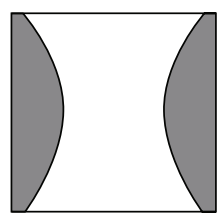

(d) $\alpha=-0.5$

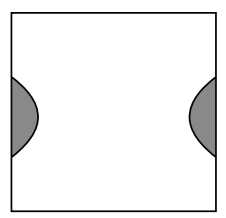

(j) $\alpha=1.1$

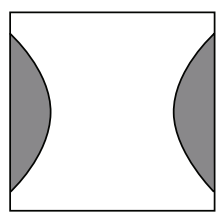

(e) $\alpha=-0.1$

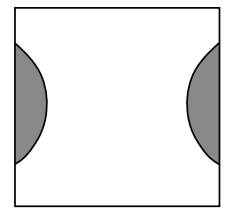

(k) $\alpha=2$

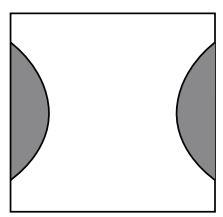

(f) $\alpha=0$

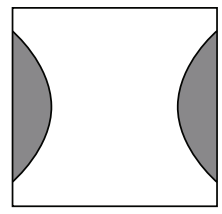

(l) $\alpha=3$

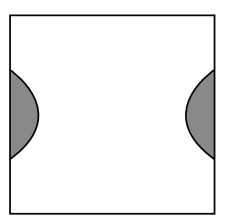

(g) $\alpha=0.5$

FIGURE 4: The drawable region of GLAC when $v=-0.01$.

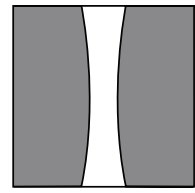

(a) $\alpha=-3$

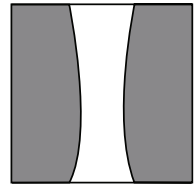

(b) $\alpha=-2$

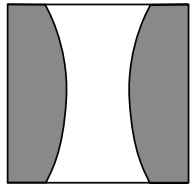

(c) $\alpha=-1$

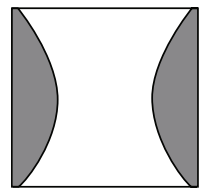

(d) $\alpha=-0.5$

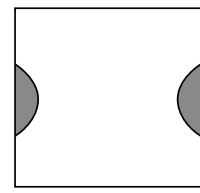

(e) $\alpha=-0.1$

FIGURE 5: The drawable region of GLAC when $v=0.01$.

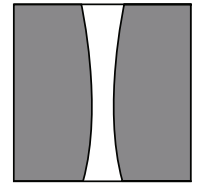

(a) $\alpha=-3$

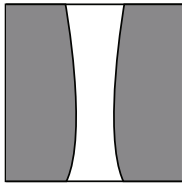

(b) $\alpha=-2$

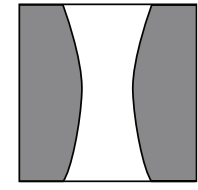

(c) $\alpha=-1$

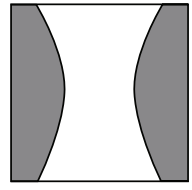

(d) $\alpha=-0.5$

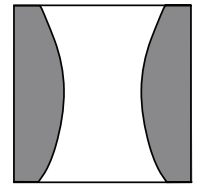

(e) $\alpha=-0.1$

Figure 6: The drawable region of GLAC when $v=0.1$.

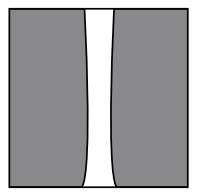

(a) $\alpha=-3$

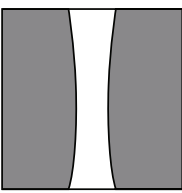

(b) $\alpha=-2$

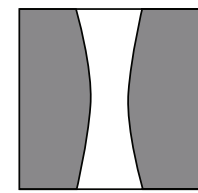

(c) $\alpha=-1$

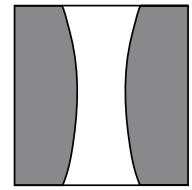

(d) $\alpha=0.5$

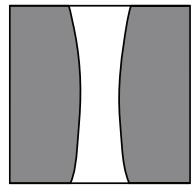

(e) $\alpha=-0.1$

FIGURE 7: The drawable region of GLAC when $v=0.3$.

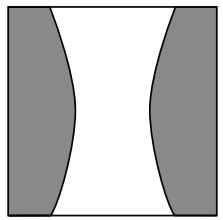

(a) $v=-0.3$

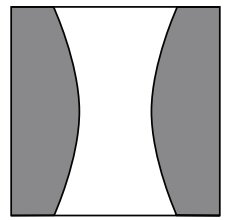

(b) $v=-0.1$

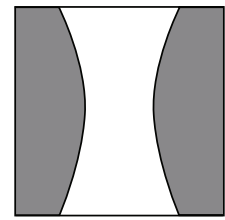

(c) $v=-0.01$

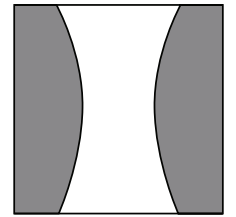

(d) $v=0.01$

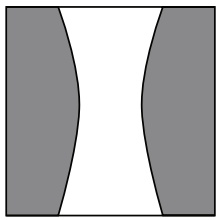

(e) $v=0.1$

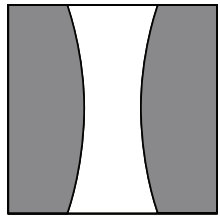

(f) $v=0.3$

FIgURE 8: The drawable region for $\alpha=-1$ as $\nu$ increases. 


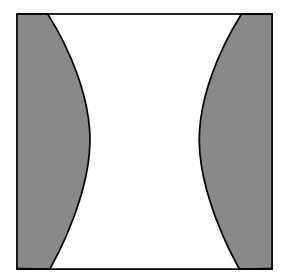

(a) $v=-0.3$

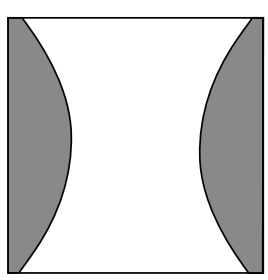

(b) $v=-0.1$

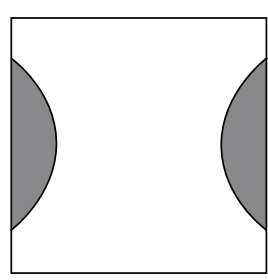

(c) $v=-0.01$

FIGURE 9: The drawable region for $\alpha=0$ as $\nu$ increases.

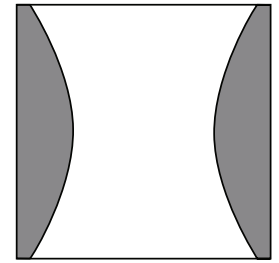

(a) $v=-0.3$

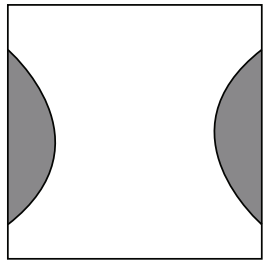

(b) $v=-0.1$

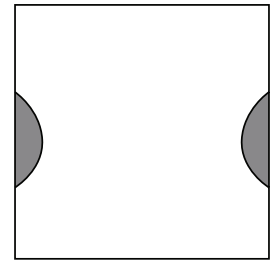

(c) $v=-0.01$

FIGURE 10: The drawable region for $\alpha=1$ as $\nu$ increases.

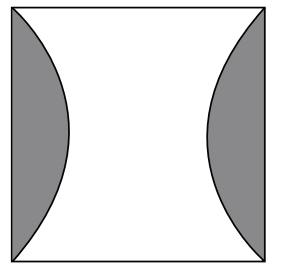

(a) $v=-0.3$

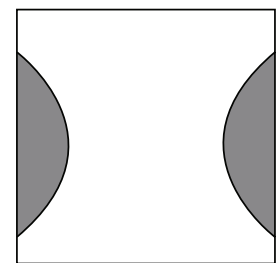

(b) $v=-0.1$

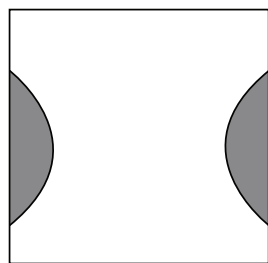

(c) $v=-0.01$

Figure 11: The drawable region for $\alpha=2$ as $\nu$ increases.

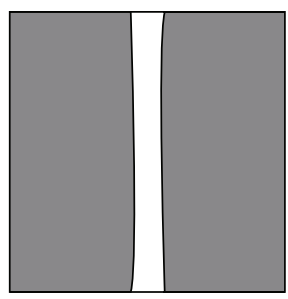

(a) $\operatorname{GLAC}(\nu=0)$

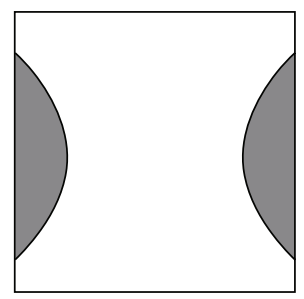

(b) $\operatorname{GLAC}(\nu=-0.01)$

FIGURE 12: Comparison on the drawable region of LAC and GLAC for $\alpha=3$.

Figure 12 is the comparison on the drawable region of LAC and GLAC when $\alpha=3$. It portrays well the role of $\nu$ in GLAC. Note that GLAC becomes LAC when $v$ is 0 . Since LAC is one of the cases in GLAC, the numerical example above clearly shows that for $\alpha>1$, GLAC has a wider solution compared to LAC. Hence, GLAC provides greater flexibility where users would be able to create various shapes with a fixed start and end interpolating points.

\section{Conclusion and Future Works}

An algorithm has been proposed to elucidate possible solutions that exist for interactive control of GLAC segment and the result is promising. The drawable region of GLAC proved to be far greater than LAC. This gives more freedom for designers to choose control points, tangent angle, and suitable shape parameters in order to design visually pleasing industrial products. The extra shape parameter $v$ facilitates the ability to set the curvature radius at the origin which makes the GLAC more flexible.

Future works include the development of a $G^{2}$ algorithm for GLAC by manipulating the shapes variables similar to the work proposed in $[13,14]$. A different kind of approach will be redefining the curvature function in such a way that a curvature continuous Log Aesthetic Spline (LAS) can be created. 


\section{Acknowledgment}

The authors acknowledge Faculty of Science and Technology, University Malaysia Terengganu (GGP grant) and Ministry of Higher Education Malaysia (FRGS: 59265) for providing financial aid to carry out this research.

\section{References}

[1] S. Pugh, Total Design, Addison-Wesley Publishing Company, Great Britain, UK, 1991.

[2] H. W. Guggenheimer, Differential Geometry, McGraw-Hill, New York, NY, USA, 1963.

[3] N. Yoshida and T. Saito, "Interactive aesthetic curve segments," Visual Computer, vol. 22, no. 9-11, pp. 896-905, 2006.

[4] K. T. Miura, R. Shirahata, S. Agari, S. Usuki, and R. U. Gobithaasan, "Variational formulation of the log-aesthetic surface and development of discrete surface filters," Computer-Aided Design and Applications, vol. 9, no. 6, pp. 901-914, 2012.

[5] R. Ziatdinov, N. Yoshida, and T.-w. Kim, "Analytic parametric equations of log-aesthetic curves in terms of incomplete gamma functions," Computer Aided Geometric Design, vol. 29, no. 2, pp. 129-140, 2012.

[6] K. T. Miura, D. Shibuya, R. U. Gobithaasan, and S. Usuki, "Designing Log-aesthetic Splines with G2 continuity," ComputerAided Design and Applications, vol. 10, no. 6, pp. 1021-1032, 2013.

[7] J. M. Ali, R. M. Tookey, J. V. Ball, and A. A. Ball, “The generalised Cornu spiral and its application to span generation," Journal of Computational and Applied Mathematics, vol. 102, no. 1, pp. 3747, 1999.

[8] R. U. Gobithaasan, R. Karpagavalli, and K. T. Miura, "Interactive control of generalized Log-Aesthetic curves',' Journal of Applied Mathematics and Computation. In press.

[9] R. U. Gobithaasan, L. P. Yee, and K. T. Miura, "A generalized log aesthetic space curve," in Proceedings of the Joint International Conference on Human-Centered Computer Environments (HCCE '12), pp. 145-149, March 2012.

[10] R. U. Gobithaasan, R. Karpagavalli, and K. T. Miura, "Shape analysis of generalized log-aesthetic curves," International Journal of Mathematical Analysis, vol. 7, no. 33-36, pp. 1751-1759, 2013.

[11] R. U. Gobithaasan, R. Karpagavalli, and K. T. Miura, "Interactive controlof Generalized Log-Aesthetic curves".

[12] N. Yoshida and T. Saito, "The evolutes of Log-aesthetic planar curves and the Drawable Boundaries of the curve segments," Computer-Aided Design and Applications, vol. 9, no. 5, pp. 721731, 2012.

[13] R. Gobithasan and J. M. Ali, "Towards G2 curve design with Timmer Parametric Cubic," in Proceedings of the International Conference on Computer Graphics, Imaging and Visualization (CGIV'04), pp. 109-114, July 2004.

[14] A. Ahmad, R. Gobithasan, and J. M. Ali, "G2 transition curve using quartic bezier curve," in Proceedings of the International Conference on Computer Graphics, Imaging and Visualisation: New Advances (CGIV '07), pp. 223-228, August 2007. 


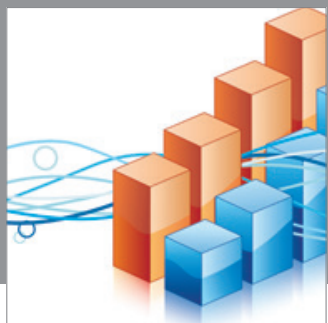

Advances in

Operations Research

mansans

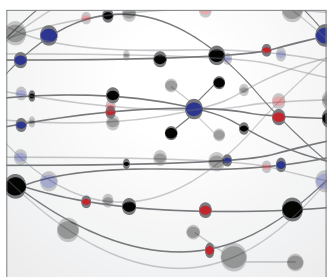

The Scientific World Journal
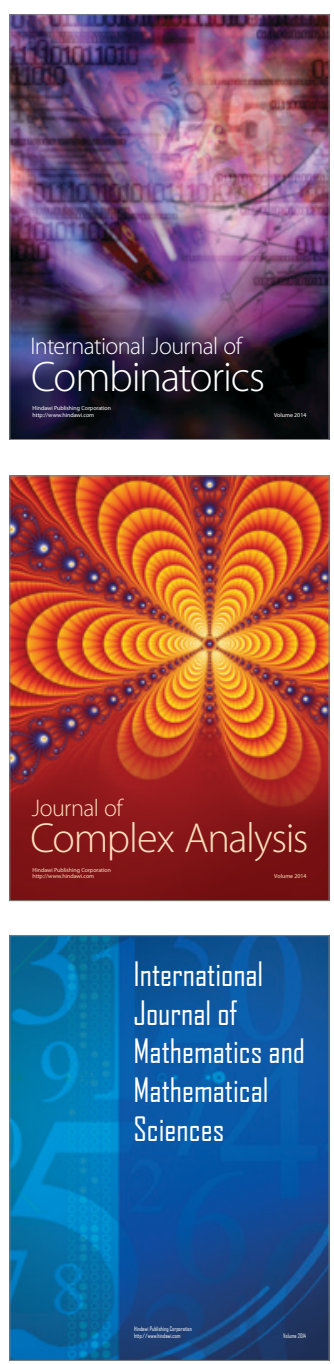
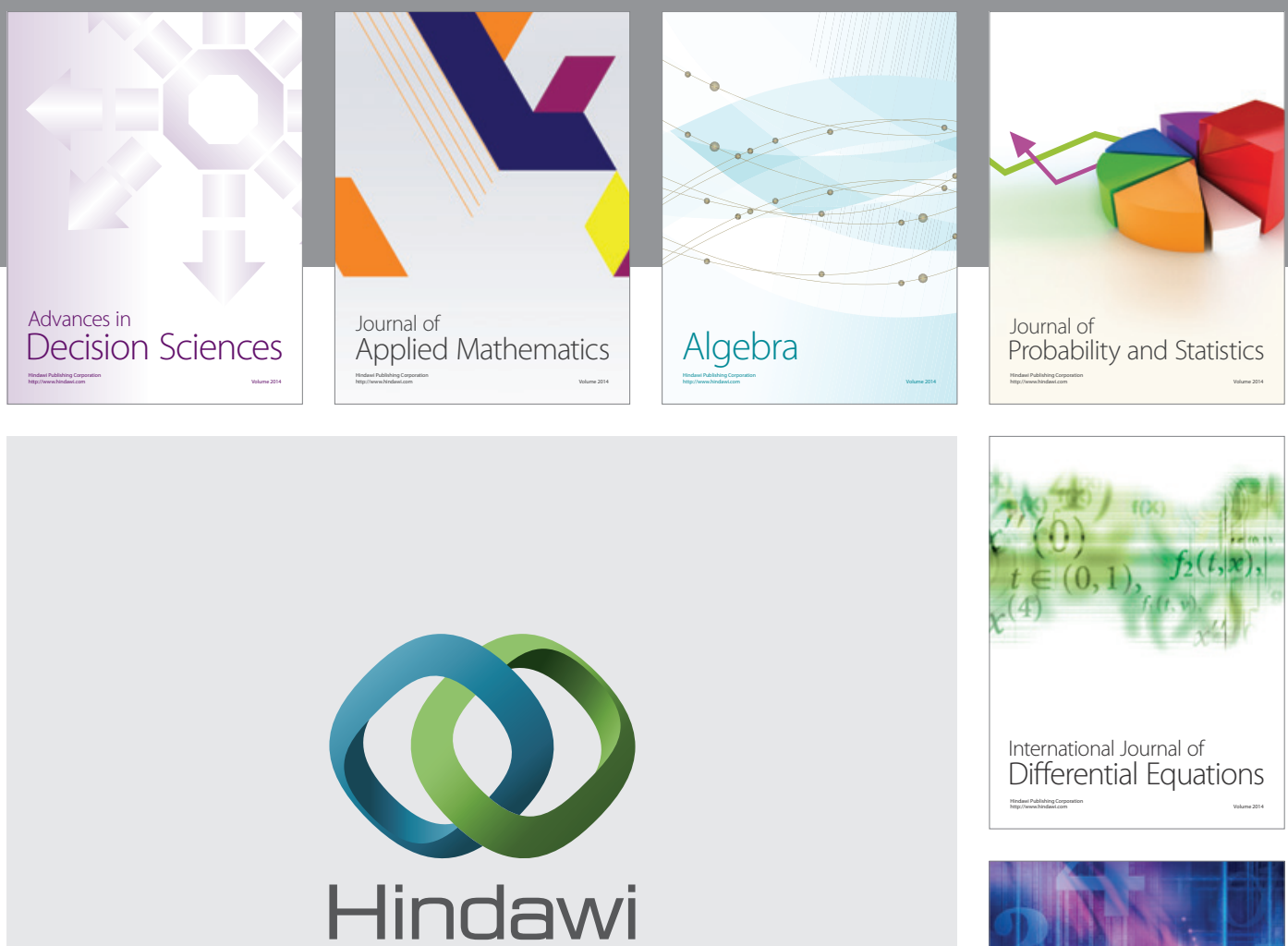

Submit your manuscripts at http://www.hindawi.com
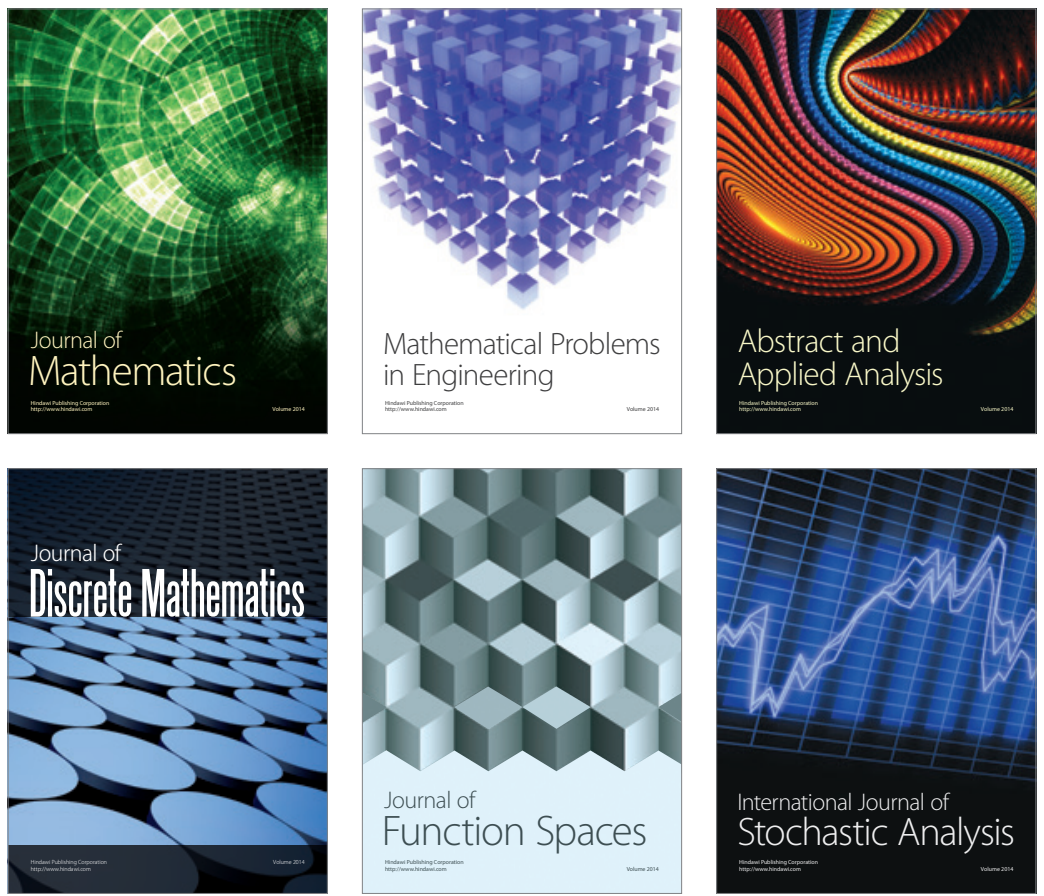

Journal of

Function Spaces

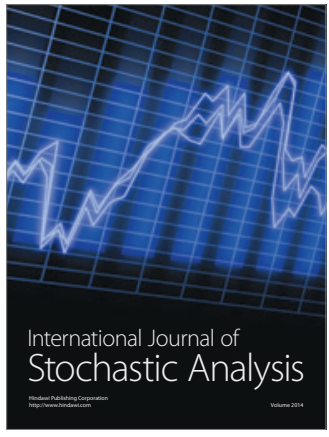

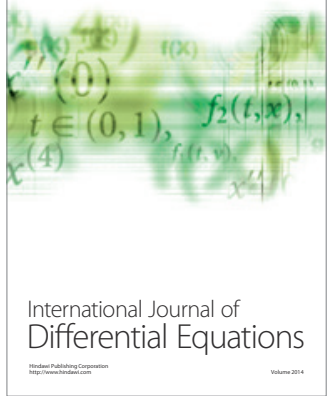
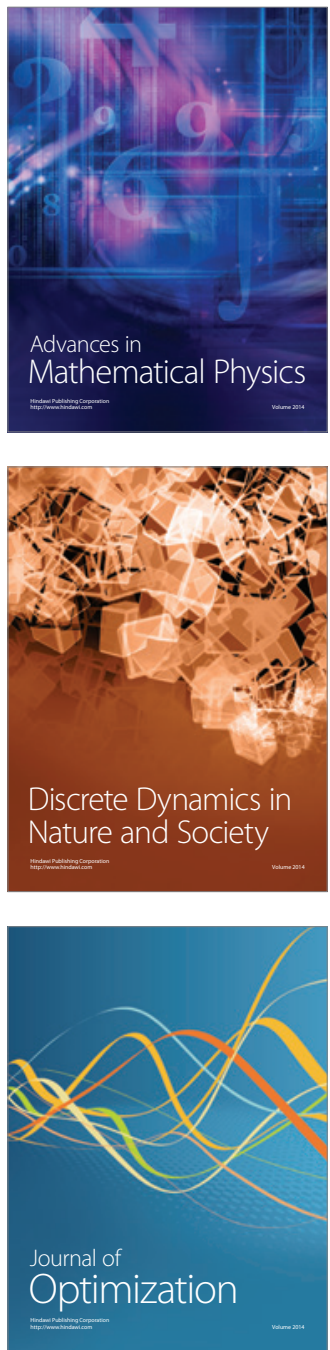